\title{
Enumeration of labeled 4-regular planar graphs
}

\author{
Marc Noy ${ }^{1}$ \\ Departament de Matemàtiques, Universitat Politècnica de Catalunya and \\ Barcelona Graduate School of Mathematics, Spain. \\ Clément Requilé ${ }^{2}$ \\ Institut für Mathematik, Freie Universität Berlin and Berlin Mathematical School, \\ Germany. \\ Juanjo Rué ${ }^{3}$ \\ Departament de Matemàtiques, Universitat Politècnica de Catalunya and \\ Barcelona Graduate School of Mathematics, Spain.
}

\begin{abstract}
In this extended abstract, we present the first combinatorial scheme for counting labeled 4-regular planar graphs through a complete recursive decomposition. More precisely, we show that the exponential generating function counting labeled 4regular planar graphs can be computed effectively as the solution of a system of equations. From here we can extract the coefficients by means of algebraic calculus. As a by-product, we can also compute the algebraic generating function counting labeled 3-connected 4-regular planar maps.
\end{abstract}

Keywords: graph enumeration, planar graphs, generating functions.

1 Email: marc.noy@upc.edu

2 Email: requile@math.fu-berlin.de

3 Email: juan.jose.rue@upc.edu 


\section{Introduction}

The enumeration of planar graphs has been subject to much research recently, see [4] and a survey in [7]. Many constrained families, such as regular planar graphs have also been studied, see for example [1] and [8] for the enumeration of cubic planar graphs.

A notable open problem is to enumerate 4-regular planar graphs. There are several references on the exhaustive generation of 4-regular planar graphs. One starts with a collection of basic graphs and shows how to generate all graphs in a certain class starting from the basic pieces and applying a sequence of local modifications. This was first done for the class of 4-regular planar graphs in [5], using as basis the graph of the octahedron. For 3-connected 4-regular planar graphs the generation was obtained in [3]. By removing isomorphic duplicates the authors were able to compute the numbers of 3connected 4-regular planar graphs up to 15 vertices. This is also the approach of the more recent reference [2] for generating quadrangulations of several types. The authors use some enumerative formulas to check the correctness of the generation procedure. However this does not include the class of 3connected quadrangulations, which by duality correspond to 3-connected 4regular planar graphs, a class for which no enumeration scheme was known.

Our main result is as follows.

Theorem 1.1 The generating functions $C^{\prime}(x)$ of labeled connected 4-regular planar graphs rooted at a vertex, where the variable $x$ encodes the number of vertices, is algebraic and expressible as a solution of a system of algebraic equations from which one can effectively compute its coefficients.

As a by-product of Theorem 1.1, one can access, up to any $n$, the $n^{\text {th }}$ coefficients of the generating functions $T(x)$ and $G(x)$ of labeled 4-regular planar graphs that are respectively 3-connected and arbitrary.

In the futur, we intend to use analytic tools to obtain precise asymptotic estimates. However the equations involved in the analysis of singularities are presently too large for this task. Some of them are polynomials of high degree in several variables with thousands of coefficients and using a standard computer algebra package we have not yet been able to perform the necessary computations. 


\section{Counting quadrangulations}

\subsection{Simple quadrangulations}

A map is rooted, so that an edge $u v$, the root edge, is distinguished and given a direction from $u$ to $v$. With this notation, $u$ will be denoted as the root vertex and the face to the right of $u v$ will be indifferently called the root face or the external face. Vertices and edges that are adjacent to the root face are also said to be external. A vertex of degree two is said to be isolated if it is not adjacent to another vertex of degree two. A quadrangulation is a map in which all faces have degree four. A diagonal in a quadrangulation is a path of length two joining opposite vertices in the root face. If $u v$ is the root edge, there are two kind of diagonals, those incident with $u$ and those incident with $v$. Note that the two kinds of diagonals cannot be present at the same time. A cycle of length four which is not the boundary of a face is called a separating quadrangle, or quad. Let $\mathcal{Q}$ be the class of simple quadrangulations, i.e. which have no multiple edge. We define the following subbfamilies of $\mathcal{Q}$ :

- $\mathcal{S}$ are irreductible quadrangulations, i.e. without diagonal and quad.

- $\mathcal{N}=\mathcal{N}_{0} \cup \mathcal{N}_{1} \cup \mathcal{N}_{2}$ are quadrangulations containing a diagonal incident with the root vertex, where $\mathcal{N}_{i}(i=0,1,2)$ are quadrangulations in $\mathcal{N}$ with exactly $i$ external isolated vertices of degree two. Clearly they are in bijection with quadrangulations containing a diagonal not incident with the root vertex.

- $\mathcal{R}$ are quadrangulations obtained from $\mathcal{S}$ by replacing each face with a quadrangulation in $\mathcal{Q}$.

For each class of quadrangulations, the corresponding generating function is written with the same letter, with variables $z$ marking internal faces and $w$ marking isolated vertices of degree two. The exception is $S(z)$, since a quadrangulation in $\mathcal{S}$ has no vertex of degree two.

Lemma 2.1 Let $\widetilde{N}=N_{0}+w^{-1} N_{1}+w^{-2} N_{2}$, then the following holds:

$$
\begin{array}{ll}
Q=z+2 N+R & N_{0}=(\tilde{N}+R)\left(\tilde{N}+R+N_{0}+(2 w)^{-1} N_{1}\right) \\
R=S(z+2 \tilde{N}+R) & N_{1}=2 z w\left(\tilde{N}+R+N_{0}+2^{-1} N_{1}\right) \\
P(z, S)=0 & N_{2}=z^{2} w^{3}+z w\left(N_{2}+2^{-1} N_{1}\right),
\end{array}
$$

where $P$ is a quadratic polynomial in $S$ (see [6]).

From the system in Lemma 2.1, we can determine a (rather long) polynomial satisfied by $Q(z, w)$. This allows us to compute $R$ and $N_{i}(i=0,1,2)$. 


\subsection{Arbitrary quadrangulations}

We define the following classes of quadrangulations:

- $\mathcal{A}_{0} \cup \mathcal{A}_{1}$ are quadrangulations of a 2 -cycle, where $\mathcal{A}_{i}(i=0,1)$ are those containing $i$ external isolated vertex of degree two.

- $\mathcal{M}=\mathcal{M}_{0} \cup \mathcal{M}_{1} \cup \mathcal{M}_{0}^{\prime}$ are arbitrary quadrangulations, where $\mathcal{M}_{i}(i=0,1)$ are those for which the root-edge is adjacent to $i$ isolated vertex of degree two, and $\mathcal{M}_{0}^{\prime}$ contains some particular cases taken out from $\mathcal{M}_{0}$.

Observe that an arbitrary quadrangulation can be obtained by replacing the edges of either the 2-path or a simple quadrangulation with quadrangulations of a 2-cycle. Let then $A=A_{0}+2 w^{-1} A_{1}$ and consider the following change of variables, that will be applied to the generating functions $N_{0}, N_{1}, N_{2}$ and $R$ : $x=z(1+A)^{2}$ and $y=\frac{w+2 A+A^{2}}{(1+A)^{2}}$. The variables $z$ and $w$ will encode the same information as before except for the generating functions $M_{0}(z, w), M_{1}(z, w)$ and $M_{0}^{\prime}(z, w)$ for which $z$ will this time mark all faces.

Lemma 2.2 Let $Q_{1}(z, w)=x^{2} y^{-1}\left(N_{1}+2 N_{2}\right)$ and $Q_{0}(z, w)=x^{2}\left(2 N_{0}+N_{1}+\right.$ $R)+\left(2 A+A^{2}\right) Q_{1}$, where $N_{0}, N_{1}, N_{2}$ and $R$ are functions of $x$ and $y$. Then the following holds:

$$
\begin{aligned}
A_{1}= & z w\left(1+A_{0}\right)(1-z(w+1))^{-1} \\
A_{0}= & 2 z A\left(1+A_{0}+A_{1}\left(1+w^{-1}\right)\right) \\
& +z\left(Q_{0}+Q_{1}\right)+z^{2}\left((1+A)^{4}+2\left(A+A^{2}\right)(w-1)\right) \\
M_{1}= & 2 z A_{1}\left(1+A_{0}+A_{1}\left(1+w^{-1}\right)\right)+z w Q_{1}+2 w z^{2} A^{2} \\
M_{0}^{\prime}= & 2 z^{2} A \\
M_{0}= & 2 z\left(1+2 A_{0}+A_{1}\left(1+2 w^{-1}\right)+\left(A_{0}+A_{1}\left(1+w^{-1}\right)\right)\left(A_{0}+A_{1} w^{-1}\right)\right) \\
& +z Q_{0}+z^{2}\left((1+A)^{4}+A^{2}(2 w-4)\right)-M_{0}^{\prime} .
\end{aligned}
$$

Now using our knowledge of $R, N_{0}, N_{1}$ and $N_{2}$ from the previous subsection, we can deduce a (very long) polynomial satisfied by $A_{0}(z, w)$ and then compute $M_{0}, M_{1}$ and $M_{0}^{\prime}$.

\section{3-connected 4-regular planar maps}

A face of degree two will be called a 2-face, and it is said to be isolated if it is not adjacent to another 2 -face. We say that an edge is ordinary if it is not in an isolated 2-face. 4-regular maps can be either rooted at an edge adacent to a 2 -face or at an ordinary edge. In this case, we call poles the two vertices adjacent to the root edge. Since the total number of edges is even, the number 
of ordinary edges is also even. We enumerate 4-regular maps according to two parameters: the number of isolated 2 -faces, marked by the variable $w$, and half the number of ordinary edges, marked by the variable $q$. When setting $w=q$ one can recover the enumeration of 4-regular maps according to half the number of edges. Observe that the dual of a quadrangulation with $\ell$ isolated vertices of degree two is a 4-regular map with $\ell$ isolated 2 -faces. We define the following families of 4-regular maps:

- $\mathcal{M}=\mathcal{M}_{0} \cup \mathcal{M}_{1} \cup \mathcal{M}_{0}^{\prime}$ are maps such that $\mathcal{M}_{i}(i=0,1)$ counts the 4-regular maps for which the root edge belongs to exactly $i$ isolated 2-face. We use the same letters as in the previous section since they are in bijections with the respective quadrangulations.

- $\mathcal{D}$ are maps whose root-edge is not counted by the variable $w$, when adjacent to a 2 -face, and counted by the variable $q$, when ordinary.

The other following families are subclasses of $\mathcal{D}$ :

- $\mathcal{D}_{2}$ are maps whose root face is an isolated 2-face and the map obtained after removing its poles remains connected. A map in $\mathcal{D}_{2}$ with poles $a$ and $b$ is typically designed to be pasted on a double edge, say $u v$, of another map by removing both double edges $a b$ and $u v$ then identifying the vertices $a$ with $u$ and $b$ with $v$.

- $\mathcal{L}$ are maps whose root edge is a loop.

- $\mathcal{S}=\mathcal{S}_{0} \cup \mathcal{S}_{1}$ are maps such that the map resulting in the suppression of the root-edge has an isthmus.

- $\mathcal{P}=\mathcal{P}_{0} \cup \mathcal{P}_{1}$ are maps such that the map resulting in the suppression of the root-edge has no isthmus but a 2 -vertex cut.

- $\mathcal{H}$ are maps obtained by taking a 3-connected 4-regular map as a core, then possibly pasting maps in $\mathcal{D}$ on its non-root edges.

Lemma 3.1 The following holds:

$$
\begin{array}{ll}
D=M_{0}+q w^{-1} M_{1}+w q^{-1} M_{0}^{\prime} & S_{1}=2^{-1} w q^{-1} L^{2} \\
L=2 q(1+D-L)+L(w+q) & P_{1}=2 w q D^{2} \\
S_{0}=D^{2}(1+D)^{-1}-q w^{-1} S_{1} & M_{0}=S_{0}+P_{0}+L+H \\
P_{0}=q^{2}\left(1+D+D^{2}+D^{3}\right)+2 q D D_{2} & M_{1}=S_{1}+P_{1}+2 w D_{2} \\
H=(1+D)^{-1} T\left(q(1+D)^{2}, w+q\left(2 D+D^{2}\right)+D_{2}\right) & M_{0}^{\prime}=2 q^{2} D
\end{array}
$$

From the knowledge of $M_{0}, M_{1}$ and $M_{0}^{\prime}$ of the previous section, we can compute $D$. Hence we can also compute $L$, then $S_{1}$ and $S_{0}$. We also know $P_{1}=2 w D^{2}$ and from $M_{1}=S_{1}+P_{1}+2 w D_{2}$ we obtain $D_{2}$. This is enough to compute $P_{0}$, and from $M_{0}=S_{0}+P_{0}+L+H$ we obtain $H$. We are ready for 
the final step. Consider the following change of variables $u=q(1+D)^{2}$ and $v=w+q\left(2 D+D^{2}\right)+D_{2}$. The first terms in the series expansion of $u(q, w)$ and $v(q, w)$ are respectively $q$ and $w$. It follows that the Jacobian at $(0,0)$ is equal to 1 and the system is invertible. Then let $q=a(u, v)$ and $w=b(u, v)$ be the inversion of the previous system. We use the last equation in Lemma 3.1 to get $T(u, v)=(1+D(a(u, v), b(u, v)) H(a(u, v), b(u, v))$. Since we already have access to $D$ and $H$, we finally have $T(u, v)$. And we can check that the coefficients of $T(u, 0)$ we obtained match those from Table 1 in [3].

\section{4-regular planar graphs}

Graph will be decomposed in a similar way as maps, following a root-decomposition of the networks, which are 4-regular planar multigraphs rooted at directed edge, such that after the removal of their root edges they become simple. We also use the same letters as in the previous section, but they now represent classes of graphs instead of maps.

We split the networks arising from a 3-connected core into the two classes $H_{1}$ and $H_{2}$, according to whether the root is simple or double. We let $T_{i}(x, u, v)$ be the generating functions of 3-connected 4-regular planar graphs rooted at a directed edge, where $i=1,2$ indicates the multiplicity of the root, $x$ marks vertices and $u, v$ are as before. The coefficients $T_{n, k, \ell}^{(i)}$ of $T_{i}$ are easily obtained from those of $T=\sum t_{k, \ell} u^{k} v^{\ell}$, the generating function of 3-connected 4-regular maps computed in the previous section. By double counting we have $T_{n, k, \ell}^{(1)}=n ! \frac{t_{k, \ell}}{2}$ and $T_{n, k, \ell}^{(2)}=\frac{k}{\ell} t_{n, k, \ell}^{(1)}, \quad$ where $\quad n=\ell+k / 2$. In terms of generating functions this amounts to $T^{(1)}(x, u, v)=\frac{1}{2} T\left(u x^{1 / 2}, v x\right)$ and $u T_{u}^{(2)}(x, u, v)=$ $v T_{v}^{(1)}(x, u, v)$, where $T_{u}$ and $T_{v}$ are the differentials of $T(u, v)$ with respect to $u$ and $v$. We observe that $D_{2}=S_{2}+H_{2}$, where $S_{2}$ counts series networks counted by $D_{2}$, i.e. the graph resulting from the removal of the two poles of a network in $\mathcal{D}_{2}$ has a cut-vertex.

Lemma 4.1 The following holds:

$$
\begin{array}{ll}
D=L+S+P+H_{1}+D_{2} & D_{2}=S_{2}+H_{2} \\
S=D(D-S) & S_{2}=x^{-1} D_{2}\left(D_{2}-S_{2}\right) \\
P=x^{2}\left(2^{-1} D^{2}+6^{-1} D^{3}\right)+2^{-1} D_{2} D & L=2^{-1} x(D-L),
\end{array}
$$


where

$$
\begin{aligned}
& H_{2}=\left(D+2^{-1} D^{2}+x^{-2} D_{2}\right)^{-1} T_{2}\left(x, 1+D, D+2^{-1} D^{2}+x^{-2} D_{2}\right) \\
& H_{1}=(1+D)^{-1} T_{1}\left(x, 1+D, D+2^{-1} D^{2}+x^{-2} D_{2}\right)+D \cdot H_{2} .
\end{aligned}
$$

These are 8 equations in 8 unknowns which in principle should allow us to determine $D$. We can finally determine $C(x)$ and $G(x)$ from the following system of equations:

$$
\begin{aligned}
& 4 C^{\bullet}(x)=D(x)-D_{2}(x)-L(x)-L(x)^{2}-x^{2} \frac{D^{2}}{2} \\
& C(x)=\int \frac{C^{\bullet}(x)}{x} d x \quad G(x)=\exp (C(x)) .
\end{aligned}
$$

\section{References}

[1] Bodirsky M., M. Kang, M. Löffler, C. McDiarmid, Random cubic planar graphs, Random Structures \& Algorithms 30 (2007), pp. 78-94.

[2] Brinkmann G., S. Greenberg, C. Greenhill, B. D. McKay, R. Thomas, P. Wollan, Generation of simple quadrangulations of the sphere, Discrete Math. 305 (2005), pp. 33-54.

[3] Broersma H. J., A. J. W. Duijvestijn, F. Göbel, Generating all 3-connected 4regular planar graphs from the octahedron graph, J. Graph Theory 17 (1993), pp. 613-620.

[4] Giménez O., M. Noy, Asymptotic enumeration and limit laws of planar graphs, J. Amer. Math. Soc. 22 (2009), pp. 309-329.

[5] Lehel J., Generating all 4-regular planar graphs from the graph of the octahedron, J. Graph Theory 5 (1981), pp. 423-426.

[6] Mullin R. C., P. J. Schellenberg, The enumeration of c-nets via quadrangulations, Discrete Math. 4 (1958), pp. 259-276.

[7] Noy M., Random planar graphs and beyond, in "Proceedings of the International Conference of Mathematicians, Seoul (2014)", vol. IV, pp. 407-430.

[8] Noy M., C. Requilé, J. Rué, Random cubic planar graphs revisited (in preparation). 